\title{
Nursing perspectives on their COVID-19 pandemic preparedness
}

Jennifer M. Moseley', Dawn Burns' ${ }^{1}$, John Heil', Katherine He ${ }^{1,2}$, Allan Stolarski ${ }^{1,3}$, Edward Whang ${ }^{1,2}$ and Gentian Kristo ${ }^{1,2^{*}}$

*Correspondence: gentian.kristo@va.gov

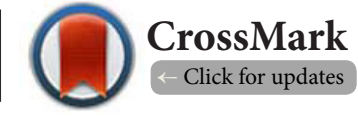

'Department of Surgery, Veterans Affairs Boston Healthcare System, Boston, MA, USA.

${ }^{2}$ Department of Surgery, Brigham and Women's Hospital, Harvard Medical School, Boston, MA, USA.

${ }^{3}$ Department of Surgery, Boston Medical Center, Boston University Medical School, Boston, MA, USA.

\begin{abstract}
Background: Nurses are essential for caring for patients during the global COVID-19 pandemic. Our objective was to explore the perspectives of nurses regarding their COVID-19 pandemic preparedness and determine areas for improvement.

Methods: An anonymous web-based survey was distributed between April 24 and May 3, 2020to 127 nurses working in medical/surgical floors at a medium size hospital in the Northeast region of the USA.

Results: A total of 79 of 127 nurses participated in our survey (62.2\% response rate). The most significant impact of the COVID-19 pandemic on our respondents' personal/family life was the concern about infecting family members with COVID-19, followed by increased stress and anxiety; lack of childcare; reduced social activities; inability to visit parents or close family members; self-isolation from family; difficulties balancing pandemic work with family dynamics; inability to care for elderly parents; fear of getting infected with COVID-19; and financial worries.
\end{abstract}

In the workplace, nurses reported increased stress and anxiety, constantly-changing instructions, fear of getting infected with COVID-19, increased workload, inadequate preparedness, lack of appreciation from leadership, and lack of proper training for redeployment.

When considering how to improve the experience of nurses for future pandemics, the most important lesson identified by our survey participants from this COVID-19 response was a better overall pandemic preparedness, followed by improved communication; adequate cross-training for deployment in different units; education on inter-professional teamwork; better support and appreciation from leadership; including nursing input on preparedness plans; maintain social distancing at all times; mental health support; considering everyone infected until testing proves otherwise; and encouraging staff to not come to work when sick.

Conclusion: Our study shows that the COVID-19 pandemic has significantly impacted nurses both professionally and personally. Nursing and hospital leadership should facilitate nurses' input on developing solutions to meet their needs both during and after crisis scenarios.

Keywords: COVID-19 pandemic; emergency preparedness; nurse wellness; nursing education

\section{Introduction}

Nurses play an integral role in the delivery of patient care during the global pandemic caused by coronavirus disease 2019 (COVID-19). As of June 6th, 2020, there are 6,804,044 cases of COVID-19 worldwide, with 1,909,077 cases being in the United States [1] COVID-19 has presented unique challenges for health- care workers; shortages of staff, personal protective equipment (PPE), test kits, and intensive care unit (ICU) beds have led to resources being stretched thin while caring for these critically ill patients across the globe. In the Lombardy region of Italy and elsewhere around the world, patients overwhelmed the available resources necessitating the Italian government to 
Moseley et al, Journal of Nursing 2020,

intervene with additional funding, staff, and other resources [2].

Healthcare workers represent a valuable resource in the fight against COVID-19. Healthcare workers on the front lines are at high risk of infection. In China, over 3,300 healthcare workers have been infected and in Italy, one-fifth of healthcare workers have been infected with COVID-19 [3]. Given the high risk of airborne transmission, nurses are at high risk of contracting the infection by performing previously simple tasks such as the application of a nasal cannula [4]. Nurses are not only at risk of infection from COVID-19, but also psychological stress and mental health disorders [5]. The psychological stress comes from fear of being infected, fear of infecting others, separation from family, and fear of dying [5]. These psychological stressors are compounded by the fact that nurses are being deployed to areas outside their normal work and comfort zones. For example, in Massachusetts, thousands of nurses have been reassigned to different roles to fill various voids creating by the virus: ICU and emergency room staff nurses, swabbing patients to test for COVID-19, or staffing field hospitals [6].

Despite the difficulties nurses are facing, nursing ingenuity has been responsible for many actions which have improved the response to COVID-19. Some of these actions include improved staffing ratios, conservation of PPE through relocating IV pumps, and ventilator controls outside patient rooms, videoconferencing, and even digital stethoscopes that can be heard outside the patient room,all with the shared goal to reduceboth PPE use and staff exposure [7].

As nurses are an integral component of the healthcare emergency response, we sought to identify their concerns related to the COVID-19 pandemic and determine areas for improvement.

\section{Methods}

A list of possible survey questions was generated from interviews with 3 nurses. Survey validity was assessed via a 30 minutes focus group session with 3 nurses. After the survey was pilot tested in a small group of 5 nurses, we modified the survey questions based on their feedback.

Using an internet-based survey tool (SurveyMonkey. com Corporation), a questionnaire was distributed to 127 randomly selected nurses working full-time in direct patient care in medical/surgical floors at a medium size hospital in the Northeast region of the USA. The survey was distributed between April 24 and May 3,2020 and was comprised of 10 questions and associated responses consisted of "best choice" answer, open response, or ranking on a 5-point Likert-scale (Supplement 1).

The survey response tool was set up such that each participant was able to respond only once to the survey. The nurses involved in the generation the survey items, validity assessment, and pilot-testing were not included in the study sample. All the data were collected in accordance with the requirements of our Institutional Review Board after an ex- emption status was obtained. Nursing union approval was also obtained for this study.

Completion of the survey was voluntary, and anonymity was ensured by not requiring any personal identifiers. Only surveys with $>80 \%$ of questions completed were included in the analysis. Results were calculated based on the number of responses received to each individual question.

\section{Results}

Over a collection period of 10 days, a response rate of $62.2 \%$ (79/127 nurses) was achieved. The characteristics of our survey participants are displayed in Table 1.

The most common household responsibility reported by the nurses participating in our study was childrearing (49.2\%), followed by caring for elderly parents (15.3\%), caring for other family members (11.9\%), and pet care (3.4\%).

The most significant impact of the COVID-19 pandemic on our respondents' personal/family life was concern about infecting family members with COVID-19 (17.9\%), followed by increased stress and anxiety (15.4\%); lack of childcare (12.8\%); reduced social activities (11.1\%); inability to visit parents or

Table 1. Characteristics of survey respondent.

\begin{tabular}{|c|c|c|}
\hline Characteristics & & N (\%) \\
\hline \multirow[t]{6}{*}{ Age group } & $25-29$ years old & $17(21.5 \%)$ \\
\hline & 30-34 years old & $10(12.7 \%)$ \\
\hline & $35-39$ years old & $8(10.1 \%)$ \\
\hline & 40-44 years old & $11(13.9 \%)$ \\
\hline & 45 years or older & $30(37.9 \%)$ \\
\hline & Not specified & $3(3.8 \%)$ \\
\hline \multirow[t]{3}{*}{ Gender } & Female & $72(91.1 \%)$ \\
\hline & Male & $6(7.6 \%)$ \\
\hline & Not specified & $1(1.3 \%)$ \\
\hline \multirow[t]{4}{*}{ Professional degree } & Registered nurse & $57(72.1 \%)$ \\
\hline & Nurse practitioner & $14(17.7 \%)$ \\
\hline & Licensed practical nurse & $6(7.6 \%)$ \\
\hline & Not specified & $2(2.5 \%)$ \\
\hline \multirow[t]{6}{*}{ Level of education } & Nursing Certificate & $4(5.1 \%)$ \\
\hline & $\begin{array}{l}\text { Associate Degree in } \\
\text { Nursing }\end{array}$ & $10(12.7 \%)$ \\
\hline & $\begin{array}{l}\text { Bachelor of Science in } \\
\text { Nursing }\end{array}$ & $38(48.1 \%)$ \\
\hline & $\begin{array}{l}\text { Master of Science in } \\
\text { Nursing }\end{array}$ & $17(21.5 \%)$ \\
\hline & $\begin{array}{l}\text { Doctor/PhD of Nursing } \\
\text { Practice }\end{array}$ & $3(3.8 \%)$ \\
\hline & Not specified & $7(8.9 \%)$ \\
\hline
\end{tabular}


close family members (10.3\%); self-isolation from family (7.7\%); difficulties balancing pandemic work with family dynamics (6.8\%); inability to care for elderly parents (3.4\%); fear of getting infected with COVID-19 (2.6\%); and financial worries (2.6\%) (Figure 1). Other concerns (9.4\%) included negative impacts such as hospitalization of family members with COVID-19, worsening of pre-existing mental health conditions, wedding postponement, cancellation of planned vacation, fear of getting laid off from work, and cancellation of college classes. However, one positive personal impact of the pandemic was the decrease in overall time spent commuting.

Nurses participating in our survey reported that their most significant coronavirus-related concern with regards to workplace workload and wellness was increased stress and anxiety (27.9\%), followed by constantly-changing instructions (14.0\%); fear of getting infected with COVID-19 (11.6\%); increased workload (11.6\%); inadequate preparedness $(9.3 \%)$; changes in the nature of the workload (7.0\%); decreased workload (5.8\%); discomfort from wearing PPE for prolonged times (4.7\%); lack of appreciation from leadership (3.5\%); lack of proper training for redeployment (2.3\%); and worries about wellness of co-workers (2.3\%) (Figure 2).

Among 48 nurses who had been redeployed to a different unit as part of the COVID-19 response, the majority (33 [68.8\%]) report feeling adequately trained and supported for their new role.

According to our respondents, the most useful change implemented during the COVID-19 pandemic that they believe should be retained permanently for their nursing work was social distancing at work (21.3\%); followed by the ability to telework (17.3\%); flexible work hours (13.3\%); use of virtual education (12.0\%); and reduction in non-essential travel (10.7\%) (Figure 3). Some other changes (12\%) that nurses felt would beneficial in the post-pandemic era include improved nurse/patient ratios; limiting inpatient visitations, increased hospital security, and improved facility cleanliness.

When considering how to improve the experience of nurses should a future pandemic occur, the most important lesson identified by our survey participants from this COVID-19 response was a better overall pandemic preparedness (46.1\%), followed by concise and effective communication (13.7\%); adequate cross-training for deployment in different units (10.8\%); education on inter-professional teamwork (3.9\%); better support from leadership (2.9\%); including nursing input on preparedness plans (2.9\%); improving staff appreciation (2.9\%); maintaining social distancing at all times (2.9\%); increased mental health support (2.0\%); keeping the facilities always clean (2.0\%); considering everyone infected until testing proves otherwise (2.0\%); and encouraging staff to not come to work when sick (2.0\%) (Figure 4).

Other lessons (5.9\%) include interventions to boost staff morale, fair distribution of nursing tasks, ensuring that non-coronavirus patients are not neglected, schedule flexibility to accommodate nurses' personal needs, and improving professionalism.

All responses were comparable when stratified by participant gender, age group, professional degree, and level of education.

\section{Discussion}

Our study found that increased stress and anxiety, both athomeand

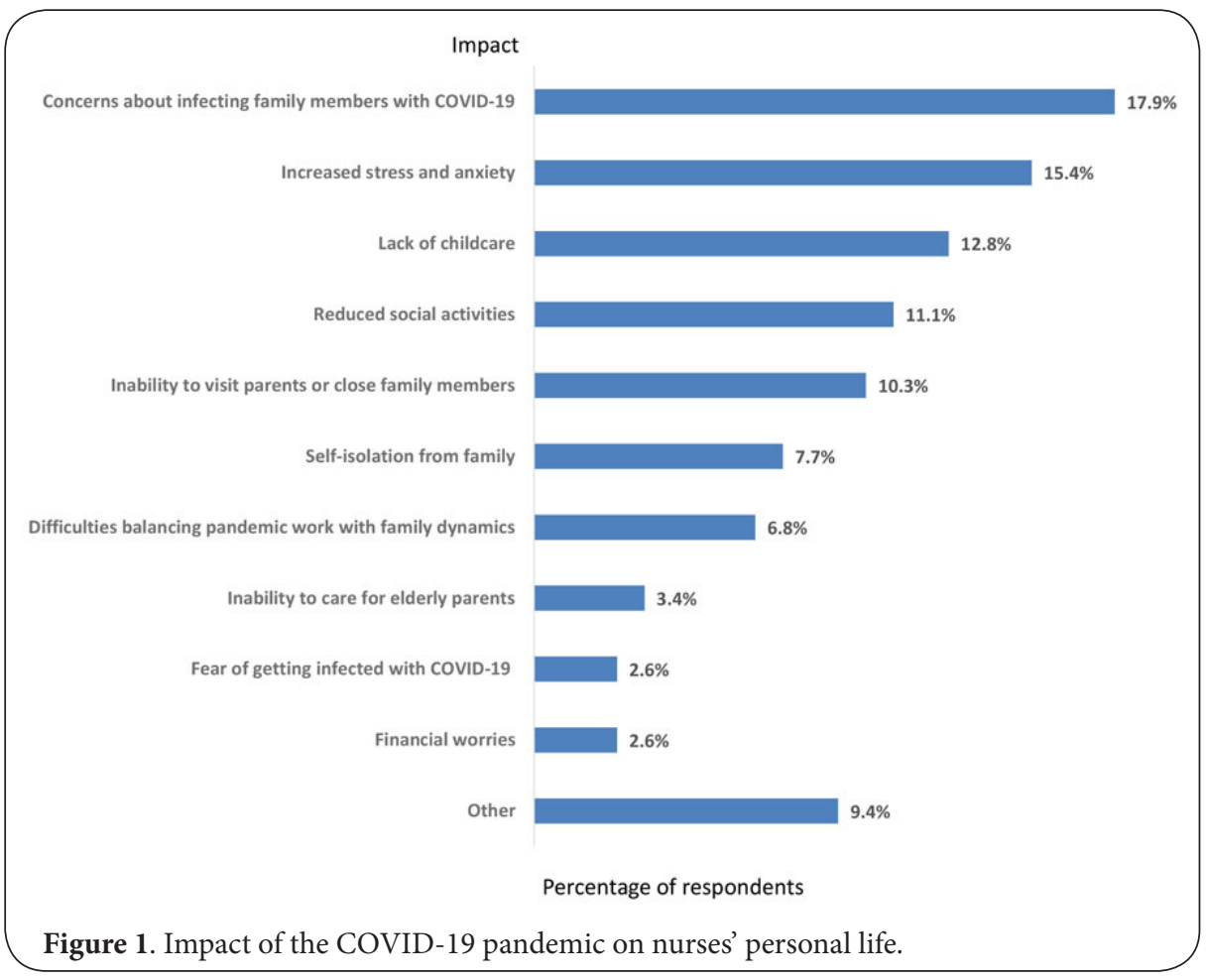


Moseley et al, Journal of Nursing 2020,

http://www.hoajonline.com/journals/pdf/2056-9157-7-3.pdf

doi: 10.7243/2056-9157-7-3
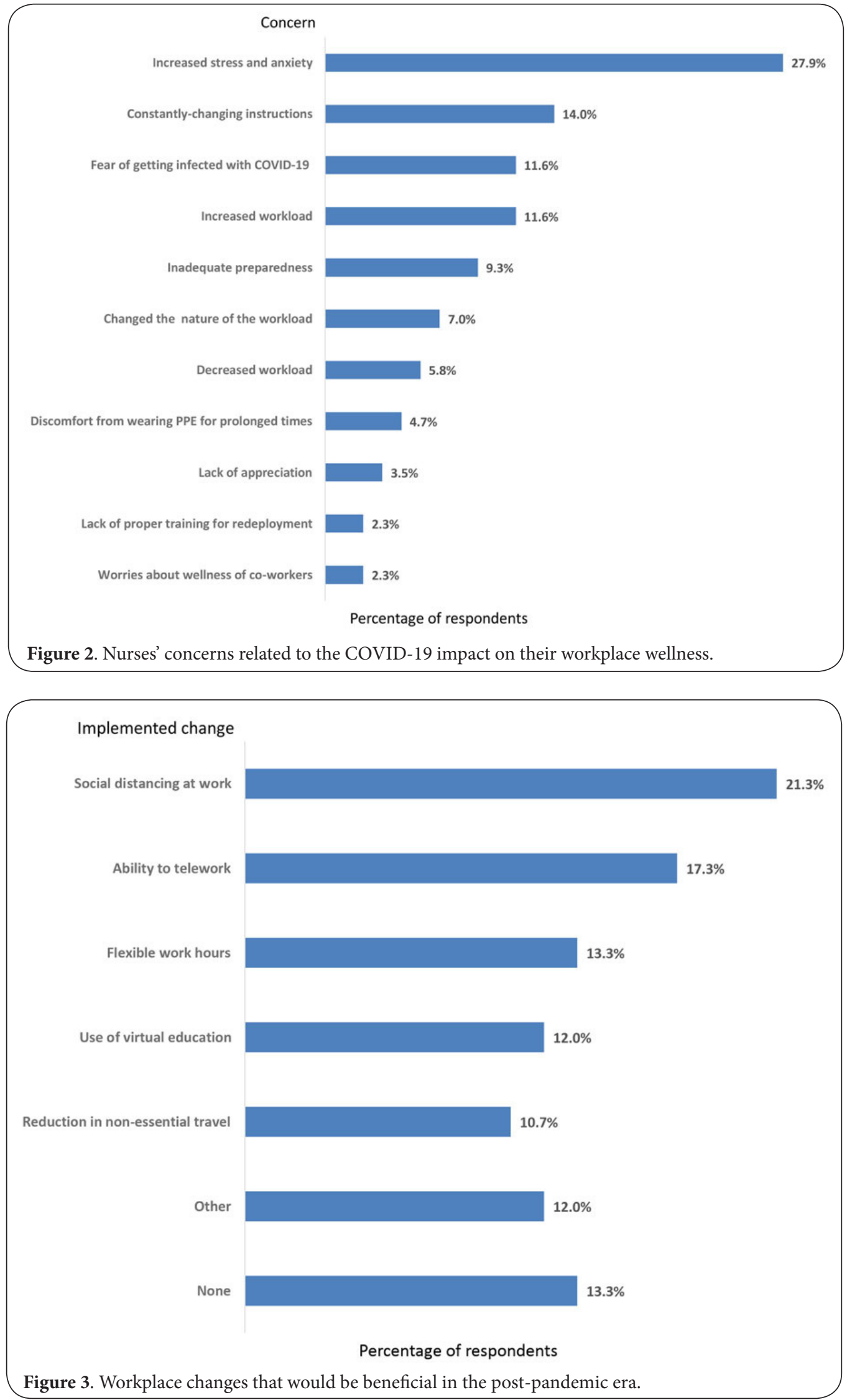
Moseley et al, Journal of Nursing 2020,

http://www.hoajonline.com/journals/pdf/2056-9157-7-3.pdf

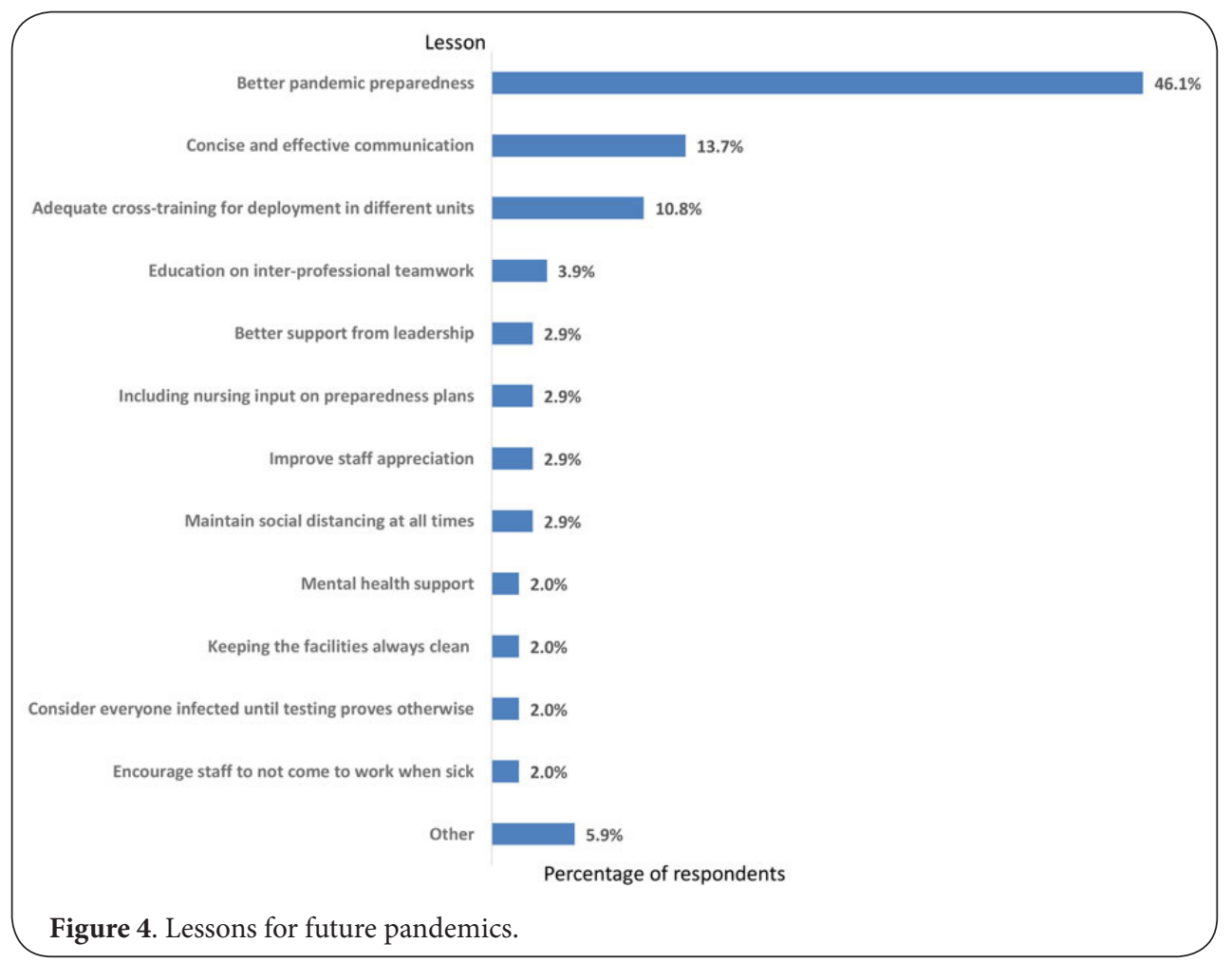

in the workplace, is the top concern among nurses. Acute and chronic stress has been linked to burnout amongst healthcare workers, which has been linked to many different disease processes including diabetes, heart disease, musculoskeletal pain, fatigue, respiratory issues, and increased mortality [8].

Experience from previous global pandemics shows that fatigue and psychosocial stress are among the most common risks to the safety and health of medical personnel in emergencies. Furthermore, healthcare workers remain at high risk for chronic post-traumatic stress disorder (PTSD) $[\mathbf{9 , 1 0 ]}$.

During the severe acute respiratory syndrome (SARS) outbreak, it was found that healthcare workers had elevated stress levels one year following the outbreak [11]. In a study done following the 2015 Middle East respiratory syndrome (MERS) outbreak, healthcare workers working with MERS patients were at risk for PTSD symptoms. These providers were especially at increased risk if they also had predisposing factors such as inadequate support system or comorbid psychiatric disorders [12]. PTSD puts nurses at risk for life-long psychological impact including increased risk for suicide.

Reports of a physician suicide related to the COVID-19 crisis have been publicized. The physician, an emergency room provider caring for COVID-19 patients,committed suicide April 2020. Her family cited stress relating to providing care for COVID-19 patients as the cause of her death [13]. Fortunately, this is the only reported case of a US healthcare worker committing suicide thus far; however, the mental health impact of this crisis is not adequately determined and must be addressed in order to prevent more needless deaths. Therefore, it is important for healthcare institutions to create professional psychological support systems to provide their workers first aid for acute stress [14]. Crisis hotlines have proved very useful for the psychological support of health care workers during the COVID-19 outbreak in China [15].

Furthermore, it is crucial to continue specialized counseling and professional psychological support even after the crisis. It is clear from past outbreaks that the impact stretches much further than the immediate crisis and long-term plans must be developed to address employee stress and anxiety. Dutheil et al. [16] recently published an article considering PTSD as the second wave of the SARS-COV-2 pandemic. They recommend taking the risk of PTSD and suicide into account when creating new healthcare policies related to COVID-19.

Employee wellness programs have been identified with improvement in stress, happiness and overall life satisfaction [17]. Thoughtfully developed wellness programs are warranted for all employees, especially nursing staff. These programs should be accessible to staff on all shifts and participation should be encouraged by management. In this period of social distancing, many nurses may feel isolated which may contribute to stress and anxiety. Social support has been identified as a way to reduce stress and anxiety levels and promote self-efficacy amongst staff treating patients with COVID-19 [18]. Self-efficacy refers to individual judgement on ability to complete a certain task, and helps individuals cope with high-intensity work while maintaining a stable mental 
Moseley et al, Journal of Nursing 2020,

state. These wellness programs should encourage virtual social support, given the constraints of social distancing. Since PTSD does not always become immediately apparent, it would be important to create long-term mental health services for nursing staff.

The status of a healthcare provider's home environment also plays a role in their stress level. Almost half of nurses identified childcare as a household responsibility. The current closures of daycare and schools has exacerbated childcare needs at home, and the impact of school and daycare closures may extend past the resolution of the first surge in COVID-19 patients. As cited in the media, the reopening status of many daycare facilities for children remains uncertain, which puts further stress on nurses to work towards finding long-term solutions for childcare [19]. The hospital administration could help during this time by providing flexible schedules, the availability to telework, and providing expanded on-site emergency childcare. These would go a long way to help alleviate the childcare concerns for these nurses.

The survey identified other lessons which can be applied to future pandemics. Nurses identified better pandemic preparedness, concise and effective communication, and crosstraining as the top three areas of how to improve. This could be accomplished through more transparency by the medical center as well as the development of standard operating procedures during pandemic scenarios leading to increased preparedness and education for all healthcare providers.

Effective communication is key during health crises. Lessons from previous pandemics have demonstrated that gaps in communication contribute to a delayed response [20]. In reviewing lessons learned from previous global epidemics, the World Health Organization(WHO) recommends risk communication with health care workers as a crucial part of the response plan during the stages of preparedness, response and recovery [14]. As recommendations during outbreaks are rapidly evolving, risk communication should be personal, face-to-face when possible, fair, and should promote a noblame culture [14].

Communication overload can be a major challenge during an emergency response as data and guidance change quickly. Experience from previous epidemics $[21,22]$ suggests that in order to prevent information overload, conflicting recommendations, or duplicated efforts, it is important for nursing leadership to provide information that is:

-Open and honest

-Empathetic

-Timely

-Concise (presented in bullet-point forms, with the main points clearly highlighted)

-Prioritizing key messages (and including additional back ground in an attached document or web link)

-Posting a version number on every guidance document including date and time stamping information

-Summarizing revisions to communications and guidance in bullets, clearly outlining changes made

Providing an avenue for staff to voice their concerns and making future pandemic plans available for all staff to review and comment provides nurses an outlet to contribute to the guidelines and operating procedures derived from their valuable first-hand experience at bedside. Continuing education programs designed to encourage interprofessional collaboration, cross-training and infection prevention should be considered. Guidance for cross training may be adapted from those recommendations for float nurses who are expected to work in a variety of environments with a potentially diverse patient population. Training competencies could be developed to ensure nurses who are floated feel adequately prepared. These should be provided to the nurses as well as guidelines for the unit they are being floated to [23]. These will contribute to increased levels of competencies when providing care and developing guidelines accessible for easy reference when they are working in novel situations. Our survey study leads us to recommend continuing this education beyond the current crisis to ensure nurses are prepared should there be a resurgence of COVID or alternative pandemic in the short or long-term.

Our study is not without limitations. First, the small sample size could limit the ability to generalize our findings. However, the total number of participating nurses is within the range of sample sizes for other studies in the nursing literature. Second, workplace differences could exist such that the workplace conditions for nurses working within our hospital could be different than for nurses working in other hospitals. However, during COVID-19, our hospital system played a crucial role of providing care to a highly vulnerable population of patients to coronavirus.

Surveying a larger group of nurses across different healthcare institutions and different specialty areas would be an important next step in generalizing and validating the findings of our study.

Third, with a response rate of $62.2 \%$, there are unaccounted perspectives, but we assume the responses collected are representative of the whole. By querying nurses from different units in an anonymous manner, we aimed to minimize both selection and recall bias, however this remains an inherit limitation to all survey studies. Despite these important limitations, we hope that our study will improve our understanding of the challenges faced by our nurses during the COVID-19 pandemic. Our findings should stimulate further discussions to identify solutions to better support our nurses both at baseline and in the setting of healthcare emergencies.

\section{Conclusions}

Nurses are essential for the response to the COVID-19 pandemic as they continue to provide dedicated patient care despite the risks to both their physical and mental health. Our study demonstrates that the COVID-19 pandemic has significantly 
Moseley et al, Journal of Nursing 2020,

impacted nurses both professionally and personally. Additionally, the findings have a broad applicability to all healthcare institutions on how to improve the experience of our nurses in the setting of healthcare emergencies and support them in providing a continued high level of care. We would advise nursing and hospital leadership to facilitate nurses' input on developing solutions to meet their needs both during and after crisis scenarios.

\section{Additional files}

\section{Supplement 1}

\section{Competing interests}

The authors declare that they have no competing interests.

\section{Authors' contributions}

\begin{tabular}{|l|c|c|c|c|c|c|c|}
\hline Authors' contributions & JMM & DB & JH & KH & AS & EW & GK \\
\hline $\begin{array}{l}\text { Research concept and } \\
\text { design }\end{array}$ & $\checkmark$ & -- & -- & -- & -- & -- & $\checkmark$ \\
\hline $\begin{array}{l}\text { Collection and/or assembly } \\
\text { of data }\end{array}$ & $\checkmark$ & -- & -- & -- & -- & -- & $\checkmark$ \\
\hline $\begin{array}{l}\text { Data analysis and } \\
\text { interpretation }\end{array}$ & $\checkmark$ & $\checkmark$ & $\checkmark$ & $\checkmark$ & $\checkmark$ & $\checkmark$ & $\checkmark$ \\
\hline Writing the article & $\checkmark$ & -- & -- & -- & -- & -- & $\checkmark$ \\
\hline Critical revision of the article & $\checkmark$ & $\checkmark$ & $\checkmark$ & $\checkmark$ & $\checkmark$ & $\checkmark$ & $\checkmark$ \\
\hline Final approval of article & $\checkmark$ & $\checkmark$ & $\checkmark$ & $\checkmark$ & $\checkmark$ & $\checkmark$ & $\checkmark$ \\
\hline
\end{tabular}

Publication history

Editor: David Reiss, Imperial College London, UK.

Received: 09-July-2020 Final Revised: 15-Aug-2020

Accepted: 30-Aug-2020 Published: 07-Sep-2020

\section{References}

1. European Centre for Disease Prevention and Control. Situation update worldwide, as of 26 April 2020.Available at:https://www.ecdc.europa.eu/ en/geographical-distribution-2019-ncov-cases. Updated 2020.Accessed 04/25/2020

2. Grasselli G, Pesenti A, Cecconi M. Critical Care Utilization for the COVID-19 Outbreak in Lombardy, Italy: Early Experience and Forecast During an Emergency Response. JAMA. Published online March 13, 2020. doi:10.1001/jama.2020.4031

3. Editorial. COVID-19: Protecting health-care workers. The Lancet. 2020;395(10228):922.

4. Ferioli M, Cisternino C, Leo V, Pisani L, Palange P, Nava S. Protecting healthcare workers from SARS-CoV-2 infection: practical indications. Eur Respir Rev. 2020;29(155):200068.

5. Petzold MB, Plag J, Ströhle A. Dealing with psychological distress by healthcare professionals during the COVID-19 pandemia. Nervenarzt. 2020;1-5.

6. McClusky P. Hospitals redeploy thousands of health care workers to respond to COVID-19 crisis. April 20, 2020. Available at: https://www. bostonglobe.com/2020/04/20/business/hospitals-redeploy-thousandshealth-care-workers-respond-covid-19-crisis/.Accessed 04/25/2020.

7. Newby JC, Mabry MC, Carlisle BA, Olson DM, Lane BE. Reflections on nursing ingenuity during the COVID-19 pandemic. J Neurosci Nurs. 2020 Mar 27. doi: 10.1097/JNN.0000000000000525. [Epub ahead of print]

8. Salvagioni DAJ, Melanda FN, Mesas AE, González AD, Gabani FL, Andrade SM. Physical, psychological and occupational consequences of job burnout: A systematic review of prospective studies. PLoS One.2017;12(10):e0185781.doi:10.1371/journal.pone.0185781.

9. Wu P, Fang $Y$, Guan $Z$, et al. The psychological impact of the SARS epidemic on hospital employees in China: exposure, risk perception, and altruistic acceptance of risk. Can J Psychiatry. 2009;54(5):302-311.

10. Sim K, Chua HC. The psychological impact of SARS: a matter of heart and mind. CMAJ. 2004;170(5):811-812.

11. Lee AM, Wong JG, McAlonan GM, et al. Stress and psychological distress among SARS survivors 1 year after the outbreak. Can J Psychiatry. 2007;52(4):233-240. doi:10.1177/070674370705200405.

12. Lee SM, Kang WS, Cho AR, Kim T, Park JK. Psychological impact of the 2015 MERS outbreak on hospital workers and quarantined hemodialysis patients. Compr Psychiatry. 2018;87:123-127.

13. Romine T. An ER doctor who continued to treat patients after she recovered from Covid-19 has died by suicide.April 28, 2020. Available at:https://www.cnn.com/2020/04/28/us/er-doctor-coronavirus-helpdeath-by-suicide-trnd/index.html. Accessed 06/02/2020.

14. Occupational health and safety of health workers, emergency responders and other workers in public health emergencies: A manual for protecting health workers and responders. Geneva: World Health Organization and International Labour Organization; 2017. Licence: CC BYNC-SA 3.0 IGO.

15. Kwai I. For China's Overwhelmed Doctors, an Understanding Voice Across the Ocean. The New York Times. Feb. 23, 2020. Available at: https:// www.nytimes.com/2020/02/23/world/asia/china-coronavirus-hotline. html. Accessed 06/02/2020.

16. Dutheil F, Mondillon L, Navel V. PTSD as the second tsunami of the SARSCov-2 pandemic [published online ahead of print, 2020 Apr 24]. Psychol Med. 2020;1-2. doi:10.1017/S0033291720001336

17. Berkland BE, Werneburg BL, Jenkins SM, et al. A Worksite Wellness Intervention: Improving Happiness, Life Satisfaction, and Gratitude in Health Care Workers. Mayo Clin Proc Innov Qual Outcomes. 2017;1(3):203-210.doi:10.1016/j.mayocpiqo.2017.09.002.

18. Xiao H, Zhang Y, Kong D, Li S, Yang N. The Effects of Social Support on Sleep Quality of Medical Staff Treating Patients with Coronavirus Disease 2019 (COVID-19) in January and February 2020 in China. Med Sci Monit. 2020;26:e923549. doi:10.12659/MSM.923549.

19. Ebbert S, Moore D. Childcare providers, parents are struggling - and worried about what comes next. May 3, 2020. Available at: https://www. bostonglobe.com/2020/05/03/metro/child-care-providers-parents-arestruggling-worried-about-what-comes-next.Accessed 06/02/2020.

20. Corless IB, Nardi D, Milstead JA, et al. Expanding nursing's role in responding to global pandemics 5/14/2018. Nurs Outlook. 2018;66(4):412-415. doi:10.1016/j.outlook.2018.06.003

21. Reynolds B, Quinn Crouse S. Effective communication during an influenza pandemic: the value of using a crisis and emergency risk communication framework. Health Promot Pract. 2008;9(4 Suppl):13S-17S. doi:10.1177/1524839908325267

22. Association of State and Territorial Health Officials. Addressing communication challenges-during an infectious disease emergency response. Available at: https://www.astho.org/Programs/InfectiousDisease/Addressing-Communication-Challenges-During-an-InfectiousDisease-Emergency-Response. Accessed 6/02/2020.

23. O'Connor K, Dugan JL. Addressing floating and patient safety. Nursing. 2017;47(2):57-58. doi:10.1097/01.NURSE.0000511820.95903.78

\section{Citation:}

Moseley JM, Burns D, Heil J, He K, Stolarski A, Whang E and Kristo G. Nursing perspectives on their COVID-19 pandemic preparedness. J Nurs. 2020; 7:3.

http://dx.doi.org/10.7243/2056-9157-7-3 